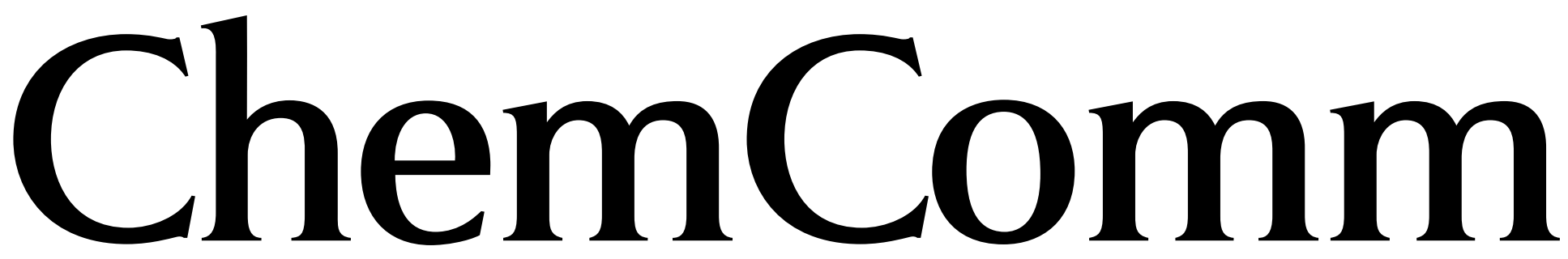

赔

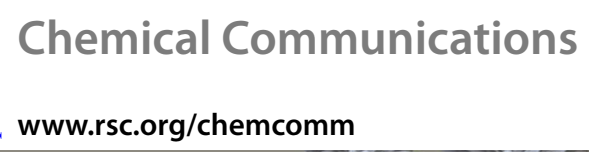

过

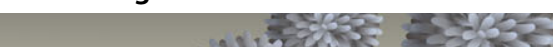




\title{
Laser desorption ionization mass spectrometric imaging of mass barcoded gold nanoparticles for security applications $\dagger$
}

\author{
Brian Creran, Bo Yan, Daniel F. Moyano, Michael M. Gilbert, Richard W. Vachet and \\ Vincent M. Rotello*
}

Received 21st January 2012, Accepted 1st March 2012

DOI: $10.1039 / \mathrm{c} 2 \mathrm{cc30499f}$

Patterns created by the inkjet printing of functionalized gold nanoparticles (NPs) can be selectively detected by laser desorption/ionization imaging mass spectrometry (LDI-IMS). These patterns can only be visualized by mass, providing a robust yet tunable system for potential anti-counterfeiting applications.

Counterfeit materials are a rapidly increasing global issue. It is estimated that illegally produced materials cost between 5 and $7 \%$ of the total annual revenue of governments and businesses. ${ }^{1}$ More troubling is the prevalence of fraudulent drugs and vaccines that endanger human health, as up to $25 \%$ of all drugs available in developing countries are counterfeit in packages created to appear as legitimate product. ${ }^{2}$ These threats are exacerbated by technological advances in image capturing and printing techniques that give criminals new tools to produce high quality copies of drug packaging, currency, and security documents.

While overt protection strategies based on physical or visual inspection remains an important part of verifying authenticity, covert methods using specialized materials and detection schemes have been devised for high value goods including currency $^{3}$ and pharmaceuticals. ${ }^{4}$ Forensic methods requiring laboratory analysis for authentication are of particular interest, as the overall complexity of observation serves as a counterfeiting deterrent. Destructive chemical analysis of the material by thin layer chromatography, ${ }^{5}$ liquid chromatography, ${ }^{6}$ and gas chromatography ${ }^{7}$ can identify the inks and pigments used in the material; these methods are however unattractive where sample preservation is needed. Furthermore, the solvent needed for analysis can often alter the integrity of the response providing unreliable results. Ambient, non-destructive analysis techniques such as infrared reflectance, ${ }^{8}$ microscope ATRinfrared spectroscopy, ${ }^{9}$ and Raman spectroscopy ${ }^{10}$ have been used to verify authenticity, although the broader use of these methods is limited due to the lack of specific chemical information, limiting the output diversity.

710 North Pleasant St, Amherst, Massachusetts, USA. E-mail: rotello@chem.umass.edu; Fax: 413-545-2058;

Tel: 413-545-4490

$\dagger$ Electronic supplementary information (ESI) available: Particle synthesis, ink formulation, and LDI-IMS procedures. See DOI: $10.1039 / \mathrm{c} 2 \mathrm{cc} 30499 \mathrm{f}$

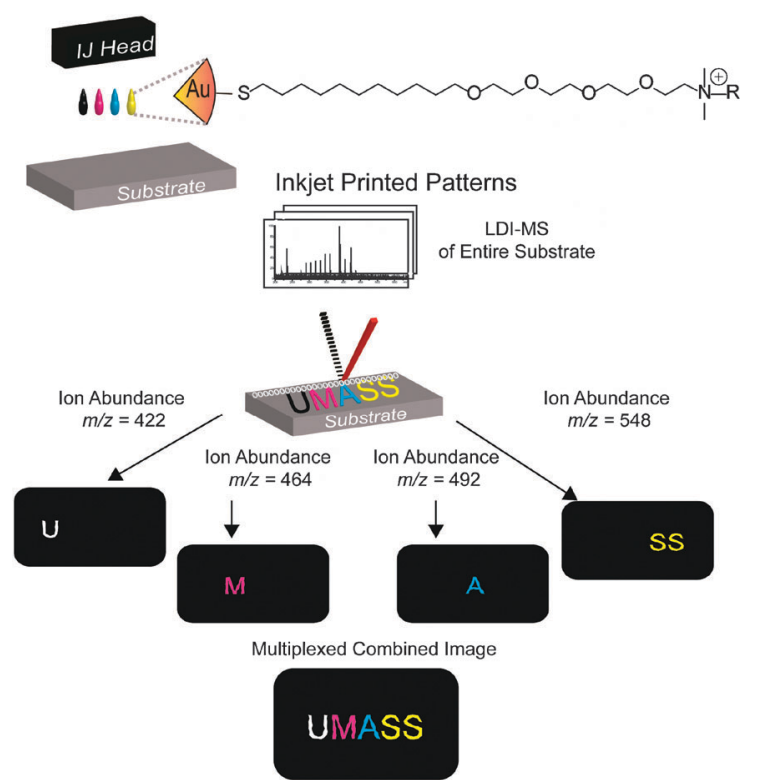

Scheme 1 Anti-counterfeiting mass barcoding strategy.

Imaging mass spectrometry (IMS) has recently been used in security applications since it produces non-destructive visual representations of mass profiles that can be compared to samples known to be genuine. ${ }^{11}$ For example, Cooks et al. used ambient IMS to analyze valid currencies against known counterfeit bills by ink analysis as a proof-of-concept verification technique. ${ }^{12}$ However, the use of solvent complicates the analysis procedure. Designing a system where a manufacturer can incorporate a specific chemical signature into their security inks whose pattern can be visualized only through IMS provides a significant challenge for the counterfeiter.

In our current research, we have demonstrated that functionalized gold nanoparticles (NPs) can be engineered for accurate detection by laser desorption/ionization MS (LDI-MS). Recent work by our groups has shown that surface ligands attached to gold NPs are ionized far more efficiently than the ligand alone due to the particle's strong absorbance at wavelengths (i.e. $337 \mathrm{~nm}$ and $355 \mathrm{~nm}$ ) commonly used in commercially available mass spectrometers. ${ }^{13}$ We report here the use of surface ligands with unique structures and mass fingerprints as "mass barcodes" to identify gold NPs (Scheme 1). These ligands can be 

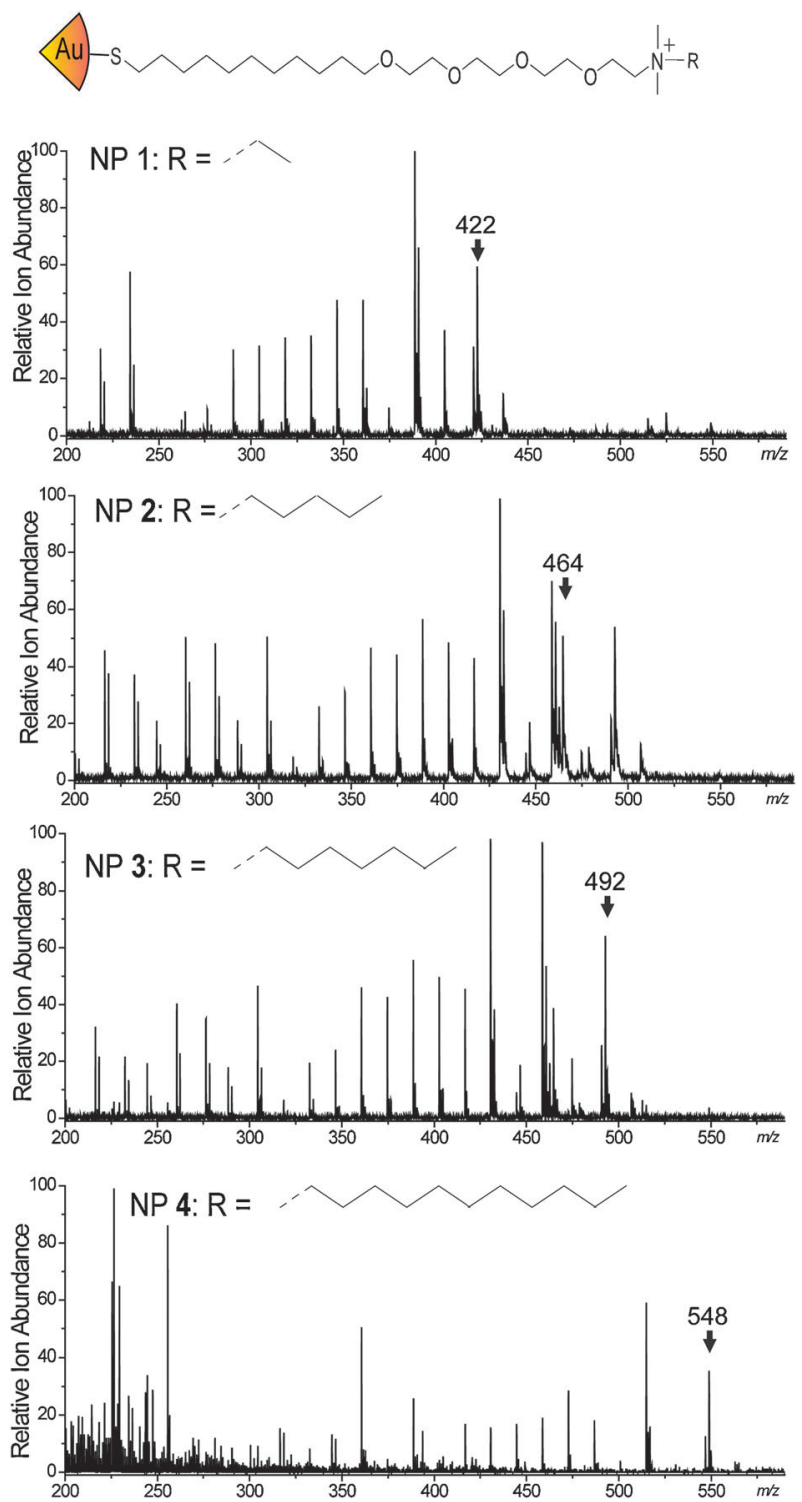

Fig. 1 Mass spectra of the four nanoparticles used in this study, with the $m / z$ value used for scanning highlighted.

altered through a wide range of synthetic means, providing flexible and tunable masses for detection by LDI-IMS. Moreover, different ligands can be employed to provide multiple channels for higher security as well as higher density of information reporting. In these studies, gold NPs were patterned onto a surface by inkjet printing. The correct pattern was visible only when the surface was scanned for the correct mass signature of the gold NPs.

The ligands for our study were chosen to achieve distinct mass fragmentation signals. The ligands featured a thiol bonding group for the gold nanoparticles, an alkane chain to stabilize the ligand shell, an oligo(ethylene) glycol to aid water solubility, and a variable ammonium group with a flexible mass head group to provide distinctive mass signatures. The chemical structures of the particles and the corresponding MS spectra are shown in Fig. 1. Gold NPs synthesis, place exchange, and inkjet ink formulation can be found in the ESI. $\dagger$

To validate our system, we first deposited gold NP 1 onto indium tin oxide (ITO) coated glass slides, commonly used in IMS. ${ }^{11 b, 14}$

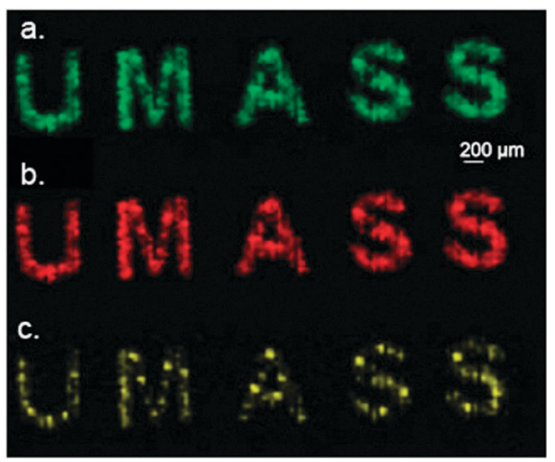

Fig. 2 (a) The $\mathrm{Au}^{+}$signal determined by scanning the ITO coated glass surface (b) the $\mathrm{Au}_{2}{ }^{+}$signal and (c) the NP 1 ligand signal (detected ions: $\mathrm{Au}^{+} m / z=197, \mathrm{Au}_{2}{ }^{+} m / z=394$, NP 1 ligand $m / z=422$ ).

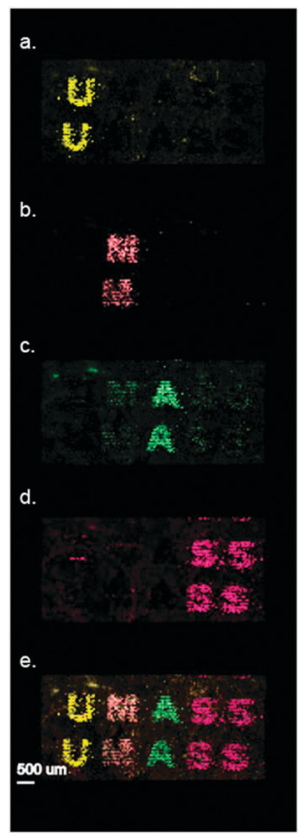

Fig. 3 Ligand LDI-IMS signals for the various ligands tinted different colors for viewing. (a) NP 1 (b) NP 3 (c) NP 2 and (d) NP 4 (e) all 4 signals combined showing the completed pattern.

The ions from the gold core as well as ions from the surface ligands were successfully detected. In Fig. 2a, we show the successful patterning of our gold nanoparticle inks as the specified pattern can be seen when analyzed for the $\mathrm{Au}^{+}$ signal. Scanning for both the $\mathrm{Au}_{2}{ }^{+}$signal and the NP 1 ligand also provided the correct image as seen in Fig. $2 \mathrm{~b}$ and $2 \mathrm{c}$, respectively.

To further investigate the capability of this inkjet printing technique, we printed separate inks in one printing cycle. A commercially available Nano Assisted Laser Desorption Ionization (NALDI ${ }^{\mathrm{TM}}$ ) surface was employed in the following experiments. ${ }^{15}$ For our study, we deposited four different gold NP inks onto the NALDI substrate to investigate their use as an anti-counterfeiting technique. Fig. 3a shows a LDI-IMS searching for just the mass barcode for NP 1, indicating no visible response from the other printed gold NP inks. Fig. 3b-d also show minimal response from the other mass barcodes, however, combining all of these scans provides us 


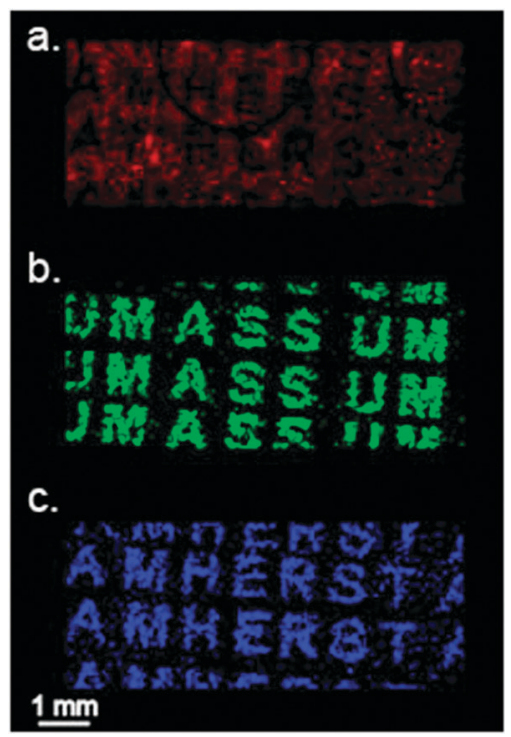

Fig. 4 Overlapped two channel printing. MSI of overlapped printing gold NPs, detected ions: blue letters AMHERST (NP $4 \mathrm{~m} / \mathrm{z}=548$ ), green letters UMASS (NP $1 m / z=422)$, red pattern $\left(\mathrm{Au}^{+}, m / z=197\right)$.

with the image of interest (Fig. 3e). This image can only be obtained by analyzing for each of the four mass barcodes, greatly reducing the ability of the counterfeiter to visualize the image.

Since the pattern above could in theory be determined simply by scanning for $\mathrm{Au}^{+}$or $\mathrm{Au}_{2}{ }^{+}$, we printed two different patterns superimposed on each other on the same NALDI substrate area. In Fig. 4a, we saw no significant visible pattern on the substrate. However, when scanning for the NP 1 ligand $(m / z=422)$, a clear pattern is detected on the surface. When scanning for the NP 4 ligand (Fig. 4c), we saw the second pattern on the substrate that was not previously visible. This demonstrates the ability of inkjet printing to deposit multiple nanoparticle patterns onto the same portion of a substrate, providing unique and sophisticated mass signatures.

In summary, we have developed an efficient security strategy using the mass signature barcode of functionalized gold nanoparticles to provide discernible patterns through LDI-IMS. By using inkjet printing, we can create surfaces that can be quickly altered either by modifying the physical pattern or by changing the functional gold NPs used. The diversity of mass options coupled with the efficiency of the "read" process makes this strategy promising for a wide variety of covert anti-counterfeiting applications.

The research was supported by the NSF IGERT (DGE0504485) to B.C. and by a seed grant from the Center for Hierarchical Manufacturing (CMMI-1025020).

\section{Notes and references}

1 T. Staake, F. Thiesse and E. Fleisch, Eur J Market, 2009, 43, 320-349.

2 F. M. Fernandez, M. D. Green and P. N. Newton, Ind. Eng. Chem. Res., 2008, 47, 585-590.

3 I. M. Lancaster and A. Mitchell, Proc. SPIE-Int. Soc. Opt. Eng., 2004, 5310, 34-45.

4 P. Aldhous, Nature, 2005, 434, 132-136.

5 (a) C. Neumann, R. Ramotowski and T. Genessay, J. Chromatogr., A, 2011, 1218, 2793-2811; (b) V. Causin, R. Casamassima, C. Marega, P. Maida, S. Schiavone, A. Marigo and A. Villari, J. Forensic Sci., 2008, 53, 1468-1473.

6 Y.-Z. Liu, J. Yu, M.-X. Xie, Y. Liu, J. Han and T.-T. Jing, J. Chromatogr., A, 2006, 1135, 57-64.

7 J. H. Bügler, H. Buchner and A. Dallmayer, J. Forensic Sci., 2008, 53, 982-988.

8 T. Trafela, M. Strlic, J. Kolar, D. A. Lichtblau, M. Anders, D. P. Mencigar and B. Pihlar, Anal. Chem., 2007, 79, 6319-6323.

9 A. Vila, N. Ferrer, J. Mantecón, D. Bretón and J. F. García, Anal. Chim. Acta, 2006, 559, 257.

10 I. Geiman, M. Leona and J. R. Lombardi, J. Forensic Sci., 2009, 54, 947-952.

11 (a) H.-W. Tang, M. Y.-M. Wong, S. L.-F. Chan, C.-M. Che and K.-M. Ng, Anal. Chem., 2011, 83, 453-458; (b) L. A. Mcdonnell and R. M. A. Heeren, Mass Spectrom. Rev., 2007, 26, 606-643; (c) L. S. Eberlin, R. Haddad, R. C. S. Neto, R. G. Cosso, D. R. J. Maia, A. O. Maldaner, J. J. Zacca, G. B. Sanvido, W. Romão, B. G. Vaz, D. R. Ifa, A. Dill, R. G. Cooks and M. N. Eberlin, Analyst, 2010, 135, 2533-2539; (d) Y. Y. Liu, X. X. Ma, Z. Q. Lin, M. J. He, G. J. Han, C. D. Yang, Z. Xing, S. C. Zhang and X. R. Zhang, Angew. Chem., Int. Ed., 2010, 49, 4435-4437.

12 D. R. Ifa, L. M. Gumaelius, L. S. Eberlin, N. E. Manicke and R. G. Cooks, Analyst, 2007, 132, 461-467.

13 (a) Z.-J. Zhu, P. S. Ghosh, O. R. Miranda, R. W. Vachet and V. M. Rotello, J. Am. Chem. Soc., 2008, 130, 14139-14143; (b) B. Yan, Z.-J. Zhu, O. R. Miranda, A. Chompoosor, V. M. Rotello and R. W. Vachet, Anal. Bioanal. Chem., 2010, 396, $1025-1035$.

14 (a) K. Chughtai and R. M. Heeren, Chem. Rev., 2010, 110, 3237-3277; (b) D. S. Cornett, M. L. Reyzer, P. Chaurand and R. M. Caprioli, Nat. Methods, 2007, 4, 828-833.

15 (a) R. Northen, O. Yanes, M.T. Northen, D. Marrinucci, W. Uritboonthai, J. Apon, S. L. Golledge, A. Nordström and G. Siuzdak, Nature, 2007, 449, 1033-1036; (b) S. Taira, Y. Sugiura, S. Moritake, S. Shimma, Y. Ichiyanagi and M. Setou, Anal. Chem., $2008,80,4761-4766$. 